\title{
Farne und Farnverwandte als Heil-, Nutz- und Zauberpflanzen: Ethnobotanische Bedeutung von Gefässkryptogamen
}

\author{
Felix Gartmann \\ Uster, Schweiz \\ Schlüsselwörter \\ Ethnomedizin · Ethnobotanik · Pteridophyten · Farne · Bärlappe · \\ Moosfarne $\cdot$ Schachtelhalme $\cdot$ Pteridologie
}

\section{Zusammenfassung}

In dieser Arbeit wird die ethnomedizinische und ethnobotanische Bedeutung von Gefässkryptogamen, d.h. Farne, Bärlappe, Moosfarne, Schachtelhalme, Brachsenkräuter und Urfarne, in verschiedenen Kulturen beschrieben. Die gegenüber Samenpflanzen artenarme Gruppe spielt weltweit als Nährpflanzen für Menschen eine kleine Rolle, doch sind die Gefässkryptogamen als Heilpflanzen recht bedeutungsvoll. Durch ihren lange Zeit unverstandenen Fortpflanzungsmechanismus ohne Blüten, Samen und Früchte wurde den Farnen und ihren Verwandten in früheren Zeiten eine gewisse Magie zugeschrieben. Diese Zauberhaftigkeit verschwand in Europa mit dem Fortschreiten der Naturwissenschaften seit der Aufklärung; in anderen Regionen der Welt konnte sie im Bewusstsein der Menschen bis heute bewahrt werden.

\section{Einleitung}

Farne und ihre Verwandten - Schachtelhalme, Bärlappe, Moosfarne und Brachsenkräuter - finden im Allgemeinen wenig Interesse beim Menschen. Diese Pflanzen fallen weder durch Farbenpracht noch durch besondere Düfte auf. Sie liefern uns keine fetthaltigen Samen und auch keine schmackhaften Früchte, ihre Blätter schmecken häufig sehr bitter, die Stiele sind mit zähem Festigungsgewebe versehen, und die Rhizome (unterirdisch wachsende Sprossabschnitte) enthalten oft Giftstoffe. Daher erstaunt es wenig, dass die Gefässkryptogamen, wie

Überarbeitete und aktualisierte Version einer Projektarbeit im Rahmen des CAS «Ethnobotanik und Ethnomedizin» an der Universität Zürich, Schweiz.

\author{
Keywords \\ Ethnomedicine · Ethnobotany · Pteridophytes · Ferns · Clubmosses · \\ Spikemosses · Horsetails · Pteridology
}

\section{Summary}

Ferns and Fern Allies as Medicinal, Economic and Magic Plants: The Ethnobotanical Importance of Vascular Cryptogams

In this paper, the ethnomedical and ethnobotanical importance of vascular cryptogams, i.e. ferns, clubmosses, spikemosses, horsetails, quillworts, and whisk ferns, in various cultures is described. Compared to seed plants, this group lacks the abundance in species and is of little importance as edible plants for humans; however, the vascular cryptogams are of a certain significance as medicinal plants. As the reproductive mechanism without flowers, seeds, and fruits has been misunderstood for a long time, a certain magic was associated with ferns and their allies. This connotation disappeared in Europe with the progress of natural sciences since the age of Enlightenment. In other regions of the world, however, ferns are still considered as having supernatural qualities.

diese Pflanzengruppe auch genannt wird, selten ausserhalb der Pteridologie - der Farnkunde - Beachtung finden (Tab. 1). Höchstens wickelt uns die Floristin noch «etwas Grün» um den hübschen Blumenstrauss und meint damit die Wedel von Farnen. Aus diesem Grund wird hier die Frage gestellt, ob Farnpflanzen wirklich diese ethnobotanische Mauerblümchenexistenz spielen oder ob sie doch zu unterschiedlichen Zeiten und Orten als Nahrungs-, Heil- und Zauberpflanzen genutzt wurden und werden. Diese Arbeit behandelt die Biologie der Gefässkryptogamen, gibt eine kurze systematische Übersicht und beschreibt die Entwicklung des Wissens über Farne in Europa und die damit einhergehende verblassende Zauberhaftigkeit dieser blütenlosen Pflanzen. Im Hauptteil werden für jede der Grossgruppen einige ihrer Verwendungsarten als Nahrungsmittel, Heil- und Zauberpflan-

\begin{tabular}{ll}
\hline KARGER & @ 2015 S. Karger GmbH, Freiburg \\
& $1015-0684 / 15 / 0272-0100 \$ 39.50 / 0$ \\
Fax +497614520714 & \\
$\begin{array}{l}\text { Information@Karger.com } \\
\text { www.karger.com }\end{array}$ & $\begin{array}{l}\text { Accessible online at: } \\
\text { www.karger.com/szg }\end{array}$
\end{tabular}




\begin{tabular}{|c|c|}
\hline Antheridium & männliches Geschlechtsorgan auf dem Prothallium, produziert begeisselte Spermien \\
\hline Archegonium & weibliches Geschlechtsorgan auf dem Prothallium, enthält eine Eizelle \\
\hline Bischofsstab & sich ausrollendes Farnblatt, siehe Fiddlehead \\
\hline Diploid & doppelter Chromosomensatz der Sporophyten \\
\hline Farnverwandte & Schachtelhalme, Bärlappe, Moosfarne, Brachsenkräuter und Urfarne \\
\hline Fiddlehead & sich ausrollendes Farnblatt, erinnert an die Schnecke bei einer Geige \\
\hline Gametophyt & die geschlechtliche Generation der Gefässkryptogamen $=$ Prothallium mit Archegonium und Antheridien \\
\hline Gefässkryptogamen & Pflanzen mit Wurzeln und Sprossachsen, jedoch ohne Samen \\
\hline Generationenwechsel & $\begin{array}{l}\text { Wechsel zwischen der geschlechtlichen Generation (=Prothallium) und der ungeschlechtlichen } \\
\text { Generation (= Sporophyt) }\end{array}$ \\
\hline Haploid & einfacher Chromosomensatz der Gametophyten \\
\hline Hapteren & $\begin{array}{l}\text { bänderförmige Strukturen an der Oberfläche der Schachtelhalmsporen, dienen zur Fortbewegung der } \\
\text { Sporen }\end{array}$ \\
\hline Heterophyllie & sterile und fertile ( = sporenproduzierende) Blätter sind verschieden gestaltet, z.B. bei der Mondraute \\
\hline Heterospor & $\begin{array}{l}\text { Pflanzen, die kleine und grosse Sporen produzieren; aus den kleinen (= Mikrosporen) entstehen } \\
\text { männliche Prothallien, aus den grossen (= Makrosporen) entstehen weibliche Prothallien }\end{array}$ \\
\hline Homospor & Gefässkryptogamen, die nur eine Art Sporen produzieren \\
\hline Indusium & Schutzhülle über den Sori \\
\hline Kormophyten & Pflanzen, die in Sprossachse, Blatt und (häufig) Wurzel gegliedert sind \\
\hline Ligula & kleines, schleimabsonderndes Blättchen an der Basis von Blättern bei Moosfarnen und Brachsenkräutern \\
\hline Prothallium & kleines, bei den Farnen oft herzförmiges Pflänzchen mit Geschlechtsorganen (= Gametophyt) \\
\hline Pteridologie & Farnkunde \\
\hline Pteridophyten & Farne und Farnverwandte \\
\hline Rhizom & oft unterirdisch wachsender Sprossabschnitt, mit Wurzeln versehen \\
\hline Sorus, pl. Sori & Ansammlung von Sporangien, oft vom Indusium bedeckt \\
\hline Sporangium & kapselförmiges Organ, in denen die Sporen gebildet werden \\
\hline Sporen & durch den Wind verbreitete, derbwandige Zellen zur Verbreitung der Gefässkryptogamen \\
\hline Sporophyll & Blätter, auf denen Sporen gebildet werden \\
\hline Sporophyt & $\begin{array}{l}\text { diploide Generation von Gefässkryptogamen, die durch die Befruchtung der Eizellen im Prothallium } \\
\text { entstanden sind und sich durch Sporen verbreiten, deutlich grösser als die Gametophyten }\end{array}$ \\
\hline Strobilus & $\begin{array}{l}\text { zapfenförmige Anhäufung von Sporangien am Ende eines Sprosses bei Schachtelhalmen und Bärlapp- } \\
\text { gewächsen }\end{array}$ \\
\hline Widdertone & $\begin{array}{l}\text { Farne, die am Körper getragen werden und gegen Verwünschungen schützen, indem sie diese Verwün- } \\
\text { schungen dem Urheber zurückschicken }\end{array}$ \\
\hline
\end{tabular}

zen sowie weitere Anwendungen skizziert, wobei oft ein Kontinuum zwischen Essen und Heilen festzustellen ist. Es stellt sich dabei die Frage, ob es in Gebieten mit einer hohen Anzahl von Pteridophyten in der Lokalflora auch anteilsmässig viele Verwendungen der Farne und ihrer Verwandten durch den Menschen gibt.

\section{Überblick über die Gruppen der Gefässkryptogamen}

\section{Farne}

Von allen Gefässkryptogamen sind die Farne sicher die bekannteste Gruppe. Es handelt sich dabei um grüne krautige Pflanzen, die in den Tropen auch baumförmig sein können, dann aber im Gegensatz zu Laub- und Nadelbäumen keinen verholzenden Stamm haben. Die Farne haben im Vergleich zu den anderen Gruppen der Gefässkryptogamen eher grosse Laubblätter (= Wedel), an deren Unterseite sich die sporenbildenden Sporangien häufig in Gruppen zu Sori vereinigen. Die Wedel rollen sich wie Bischofsstäbe zu Beginn der Vegetationsperiode aus (Abb. 1). Die Blätter sind häufig mehrfach gefiedert und bilden Trichter. Farne sind in Europa vor allem Bewohner feuchterer Habitate wie Wälder oder Schluchten, während nur wenige Arten Felsritzen und Mauern bewohnen - wie der Zerbrechliche Blasenfarn (Cystopteris fragilis (L.) Bernh.) oder die Mauerraute (Asplenium rutamuraria L.). Die Gametophyten der allermeisten Farne sind unscheinbare, halbfingernagelgrosse, grüne, herzförmige Pflänzchen, die auf ihrer Unterseite die Geschlechtsorgane tragen.

\section{Bärlappe (Lycopodiales)}

Bärlappe (althochdeutsch für Bärentatze) (Abb. 2) sind meistens kriechende, sich gabelig verzweigende Pflanzen, die an Tannenäste erinnern. Sie besitzen kleine, schuppen- oder nadelförmige Blätter. Die Sporangien sitzen an der Basis der Blättchen, bei einigen Arten in zapfenartigen Organen abgesetzt von den vegetativen Teilen der Pflanze, z.B. beim Keulen-Bärlapp (Lycopodium clavatum $\mathrm{L}$. .). 


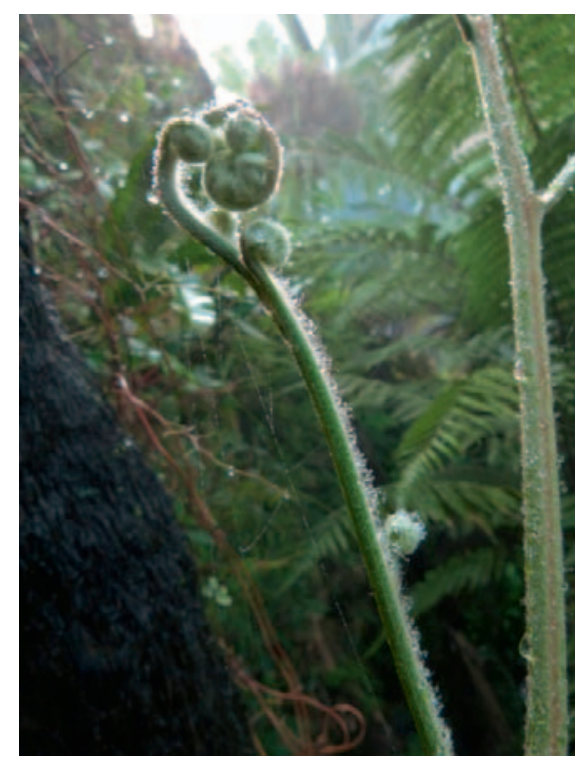

der Farnwedel (Foto:

Felix Gartmann, 2014).

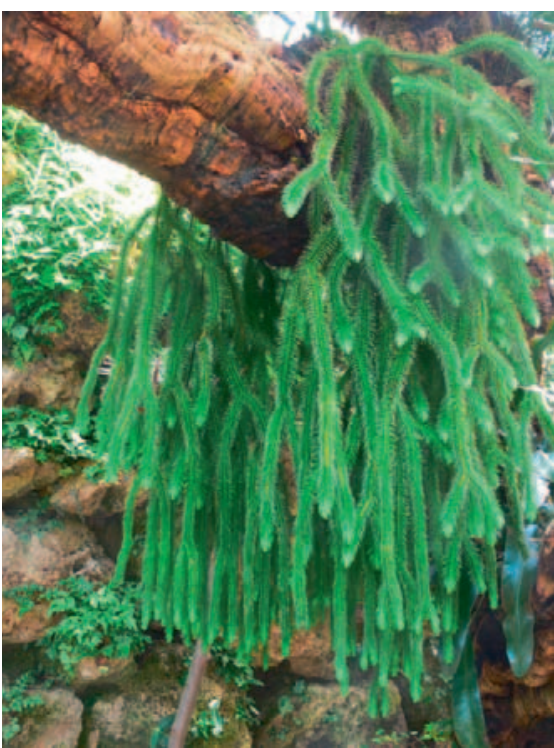

Abb. 2. Tropischer Bärlapp (Foto: Felix Gartmann, 2014).
Abb. 3. Selaginella selaginoides; unten: Selaginella Helvetica (aus Thomé OW: Flora von Deutschland, Österreich und der Schweiz. 1885).
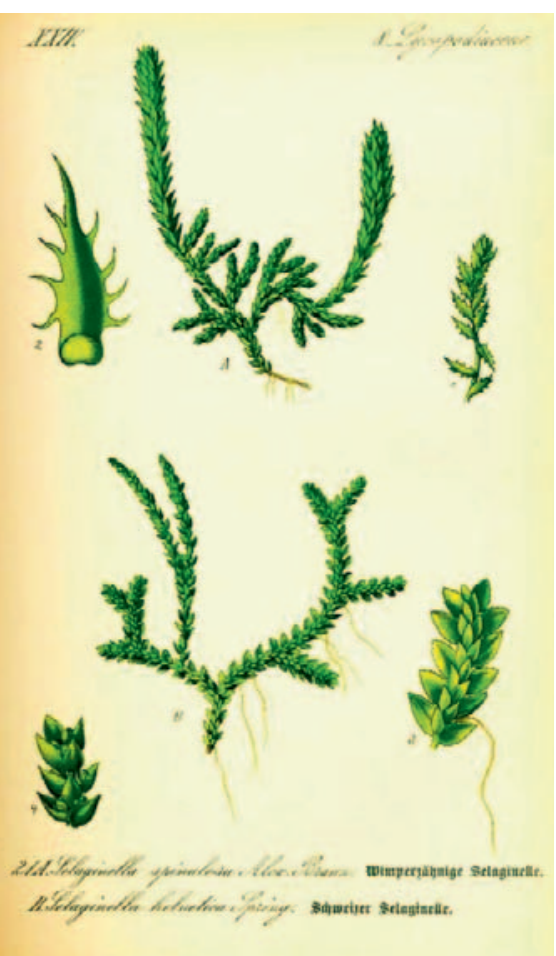

\section{Moosfarne (Selaginellales)}

Moosfarne sind eher kleine, moosähnliche heterospore Gefässkryptogamen, die in Mitteleuropa an ähnlichen Habitaten wie Bärlappe vorkommen. Trotz ihrer deutschen Bezeichnung sind Moosfarne weder Moose noch Farne, sondern am nächsten mit den Brachsenkräutern und etwas weiter entfernt mit den Bärlappen verwandt. Ihre Gametophyten sind so klein, dass sie innerhalb der Sporen bleiben. Die kleinen, schuppenförmigen Blätter der Sporophyten tragen an der Basis eine kleine Schuppe, die Ligula. Die Ordnung der Selaginellales besteht nur aus einer Familie mit der einzigen Gattung Selaginella. Sie umfasst etwa 700 Arten, wovon die meisten in den Tropen auftreten. In Mitteleuropa kommen nur Selaginella selaginoides (L.) SCHRANK \& MART. und Selaginella helvetica (L.) SPRING vor (Abb. 3).
Abb. 4. Equisetum hyemale (aus Lindman CAM: Bilder ur Nordens Flora. 1905).

\section{Schachtelhalme (Equisetales)}

Die auffälligen Schachtelhalme (die einzelnen hohlen Sprossabschnitte lassen sich ineinander schachteln) zeichnen sich durch aufrecht wachsende Kräuter aus, deren hohle Sprossen auf Höhe der kleinen, quirlig angeordneten Blätter durch Querwände in Segmente eingeteilt sind (Abb. 4). Die fertilen Pflanzen tragen endständig eine Sporangienähre, einen sogenannten Strobilus. Die Sporen enthalten Chlorophyll und weisen bewegliche, schraubig aufgewickelte Bänder an ihrer Oberfläche auf. Die Pflanzen enthalten Kieselsäure und sind schwach giftig. 
Abb. 5. Isoëtes lacustris (aus Thomé OW: Flora von Deutschland, Österreich und der Schweiz. 1885).
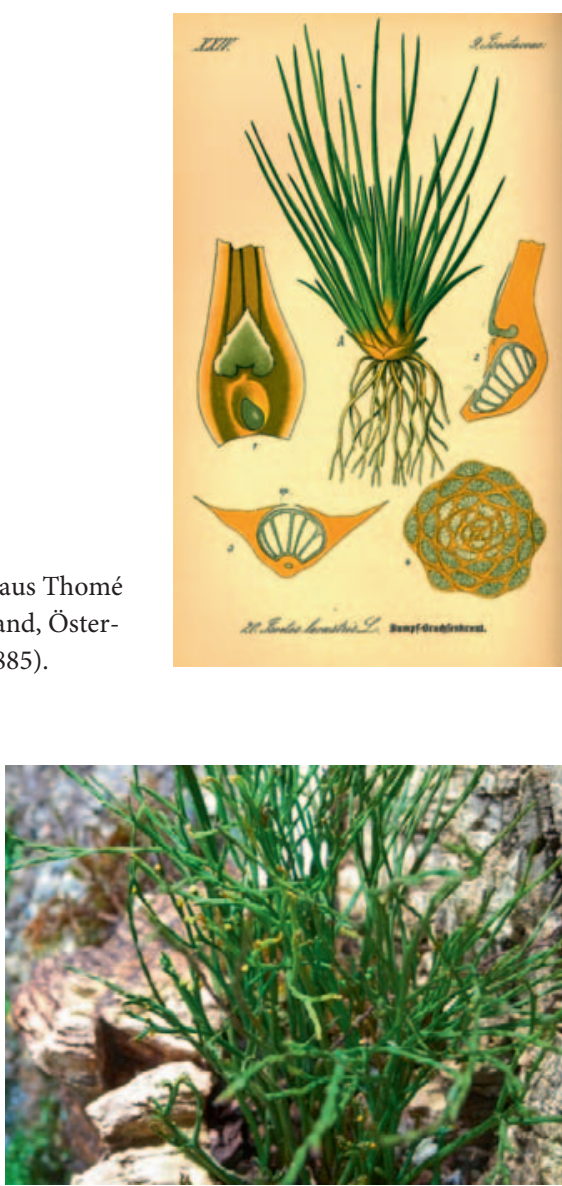

Abb. 6. Psilotum nudum (Foto: Felix Gartmann, 2014)

\section{Brachsenkräuter (Isoëtales)}

Die bei Laien kaum bekannten Brachsenkräuter (nach der Fischart Brachse, mit denen diese Pflanzen offenbar den Lebensraum teilen können) sind an Binsen erinnernde, untergetauchte heterospore Wasserpflanzen (Abb. 5). Sie stellen hohe Ansprüche an die Wasserqualität und sind daher in Europa sehr selten geworden. Die Blätter sind wie bei den Moosfarnen mit Ligulen versehen und tragen Sporangien an der Innenseite der Blätter. Die Ordnung enthält nur eine einzige Gattung mit etwa 150 Arten, wovon die meisten in den Tropen vorkommen. Die isländische Bezeichnung für das See-Brachsenkraut (Isoëtes lacustris L.) - Vatnalaukur = Wasserlauch - liefert eine gute Beschreibung dieser eigentümlichen Pflanzengruppe.

\section{Urfarne (Psilotales)}

Diese aus nur zwei Arten bestehende, urtümlich anmutende Pflanzengruppe zeichnet sich durch einen gabeligen Wuchs aus, an deren Sprossachsen winzige Blätter vorhanden sind (Abb. 6). Die auffällig gelben, kugeligen Sporangien sitzen auf den Sprossachsen. Die Pflanzen besitzen keine Wurzeln und können sich durch Brutknospen ungeschlechtlich fortpflanzen. Die gabelförmigen Gametophyten existieren unterirdisch in Lebensgemeinschaft mit Pilzen. Der einzige europäische Standort von

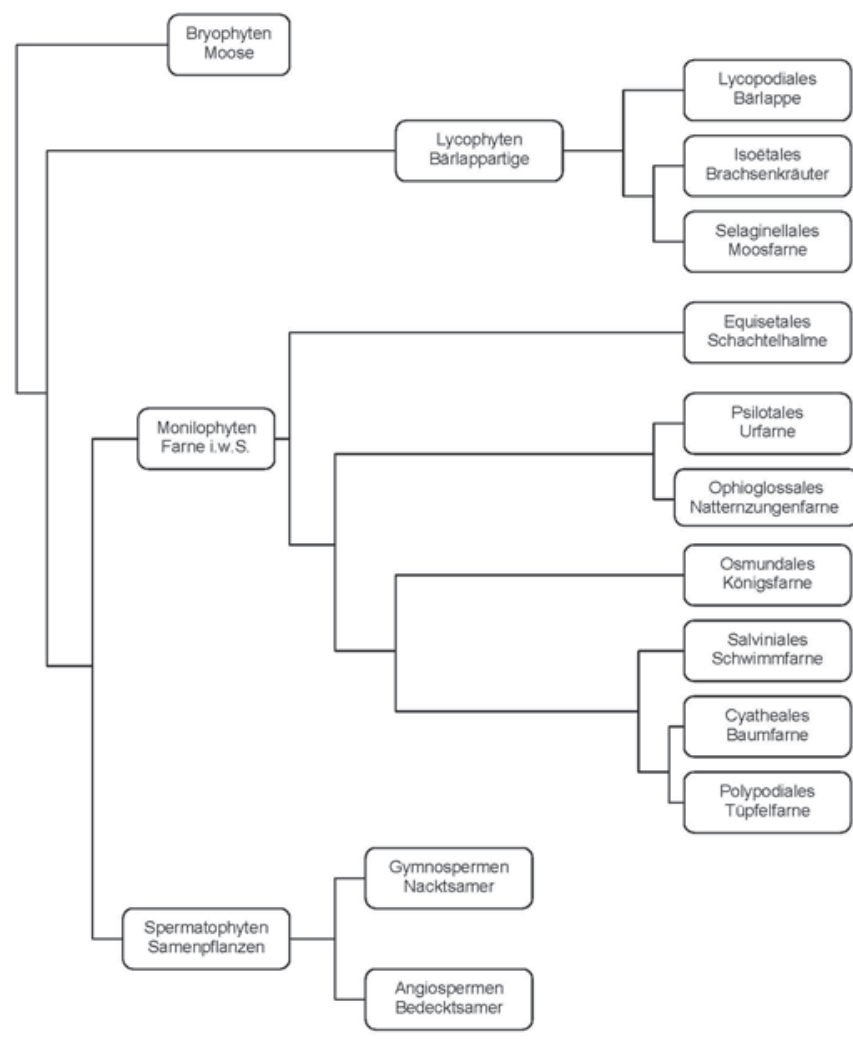

Abb. 7. Verwandtschaftsbeziehungen bei den Landpflanzen (Felix Gartmann, 2014).

Psilotum nudum (L.) P. BEAUV. liegt im Südwesten von Spanien (Montera del Torero) und ist durch die Sammeltätigkeit von Botanikern stark gefährdet [1]!

\section{Systematik der Gefässkryptogamen}

Die hier besprochenen Gruppen gehören zu den Lycophyten und Monilophyten und werden traditionell zu den Gefässkryptogamen zusammengefasst, obwohl sie nicht näher miteinander verwandt sind (Abb. 7).

Die Gemeinsamkeit dieser Pflanzen, oft auch als Farne und Farnverwandte bezeichnet, ist ihre Gliederung in Sprossachse und Wurzel sowie Blätter mit Gefässen zum Transport von Wasser und darin gelösten Stoffen; darin gleichen sie den Samenpflanzen. Im Gegensatz zu diesen produzieren Farne, Bärlappe, Moosfarne, Schachtelhalme und Brachsenkräuter aber weder Samen noch Blüten oder Früchte, was die eher bescheidene ethnobotanische Bedeutung dieser Pflanzen erklärt.

\section{Kurze Geschichte der Farnforschung}

Die Abwesenheit von Blüten, Samen und Früchten bei den Gefässkryptogamen fiel den Menschen schon früh auf. 
Abb. 8. Das pflanzliche Schaf (Quelle: Wikipedia).

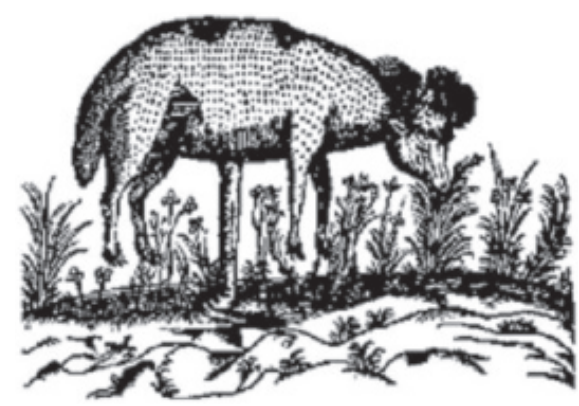

Doch was nicht zu sehen ist, muss nicht zwangsläufig inexistent sein - es kann auch schlicht unsichtbar sein. Farnsamen waren in der Vorstellung des europäischen Mittelalters und der frühen Neuzeit unsichtbar, was die Menschen zur Schlussfolgerung verleitete, dass der Verzehr dieser Samen zum richtigen Zeitpunkt den gleichen Effekt auf die Essenden hat: Man wird unsichtbar! Jedoch galten Farnsamen auch sonst als hilfreich. Legte man sie in den Geldkasten, so würde der Schatz wachsen oder doch wenigstens niemals abnehmen. Wer in der Silvesternacht Farnsamen bei sich trägt, der würde die Sprache der Tiere verstehen und könnte dadurch wertvolle Geheimnisse ergründen. Farne blühen in diesen volkstümlichen Vorstellungen nur zwischen 24 und 1 Uhr in der Johannisnacht unter Donner, Sturm und Blitzen. «Wer sie aber erlangt und nicht von sich lässt, so üblen Zauber er auch um sich herum gewahrt, der kommt zu hohen Ehren und Reichtum und erlangt die Gabe zu weissagen» [2].

Adiantum capillus-veneris L., das Frauenhaarfarn, galt bis in die frühe Neuzeit als Pflanze, die - auf dem Körper getragen - Verwünschungen einer anderen Person auf diese zurückwirft (= Widderthon oder Widdertod). Allerdings schreibt schon Bock, «das schön golt farb jungfraw hor darmit etlich zauberei treiben, nent man widderdot es haben die alte weiber vil fantasei mit disen kreüttern (...)»[3].

Auch sonst regten Farne die Fantasie unserer Vorfahren an. So geisterte das pflanzliche Schaf aus dem Land der Tartaren als Wandersage durch das ganze Mittelalter und die frühe Neuzeit. Dieses seltsame Tier sei mit dem Bauch auf einem Stamm festgewachsen und würde das Gras abfressen, das es von seiner unbequemen Position erreichen kann (Abb. 8). Sobald alles aufgefressen sei, würde es sterben. Sein «Fell» wurde damals als Verpackungsmittel und als blutstillender Verband verwendet. Möglicherweise ist damit der mit glänzenden weichen $\mathrm{Fa}$ sern versehene Baumfarn (Cibotium cumingii Kunze) (Abb. 9) gemeint [4].

In Norwegen soll die Mondraute (Botrychium lunaria (L.) Sw.) (Abb. 10), die man in Vollmondnächten erntete, mit ihren an altertümliche Schlüssel erinnernden sterilen Blattabschnitten Schlösser von Schatztruhen öffnen können [5]. Durch das Verbrennen von Adlerfarn (Pteridium

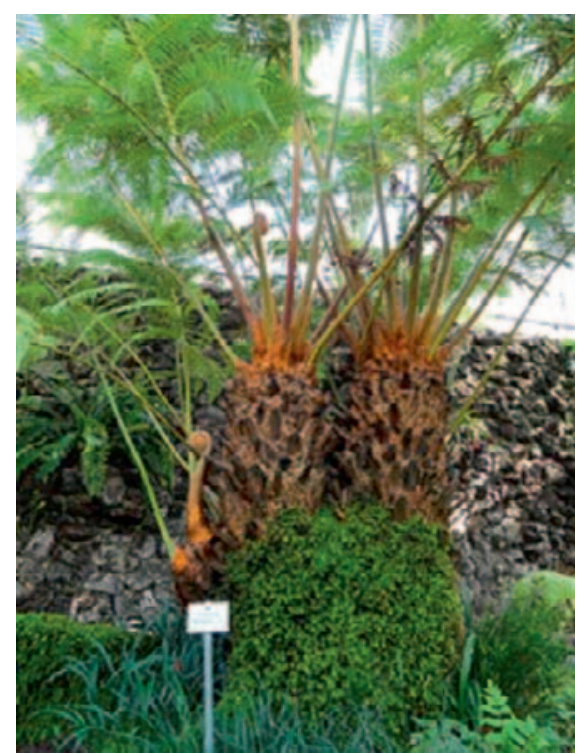

mingii

(Quelle: Wikipedia).

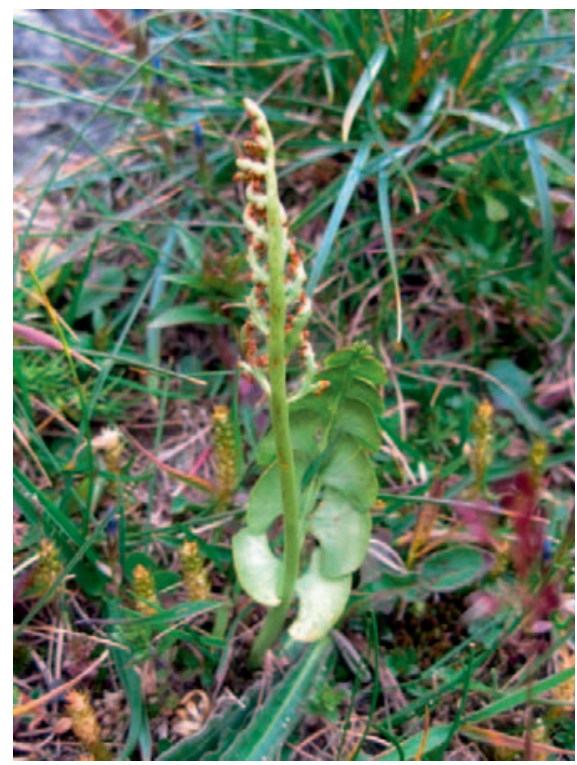

Abb. 10. Mondraute (Botrychium lunaria) (Foto: Felix Gartmann 2014).

aquilinum (L.) KUHN) sollen böse Geister ferngehalten werden [6].

Jedoch waren auch der Wissenschaft die Farne und ihre Verwandten rätselhaft. Da die Fortpflanzung der Gefässkryptogamen vor dem 19. Jahrhundert völlig unbekannt war, schloss der deutsche Renaissance-Naturforscher Leonhart Fuchs [7] von der Form auf die Funktion: Eine häufig im mitteleuropäischen Wald anzutreffende robuste Farnart musste das Männchen sein, die zartere, ebenfalls häufig vorkommende Variante betrachtete er naheliegenderweise als das Weibchen. Dieser Irrtum hat sich bis heute in der botanischen Namensgebung gehalten: Der robuste Gemeine Wurmfarn heisst Dryopteris filix-mas (L.) Schott, also Farnmännlein, der zartere Waldfarn (Athyrium filix-femina (L.) Roth.) entsprechend Farnweiblein. 


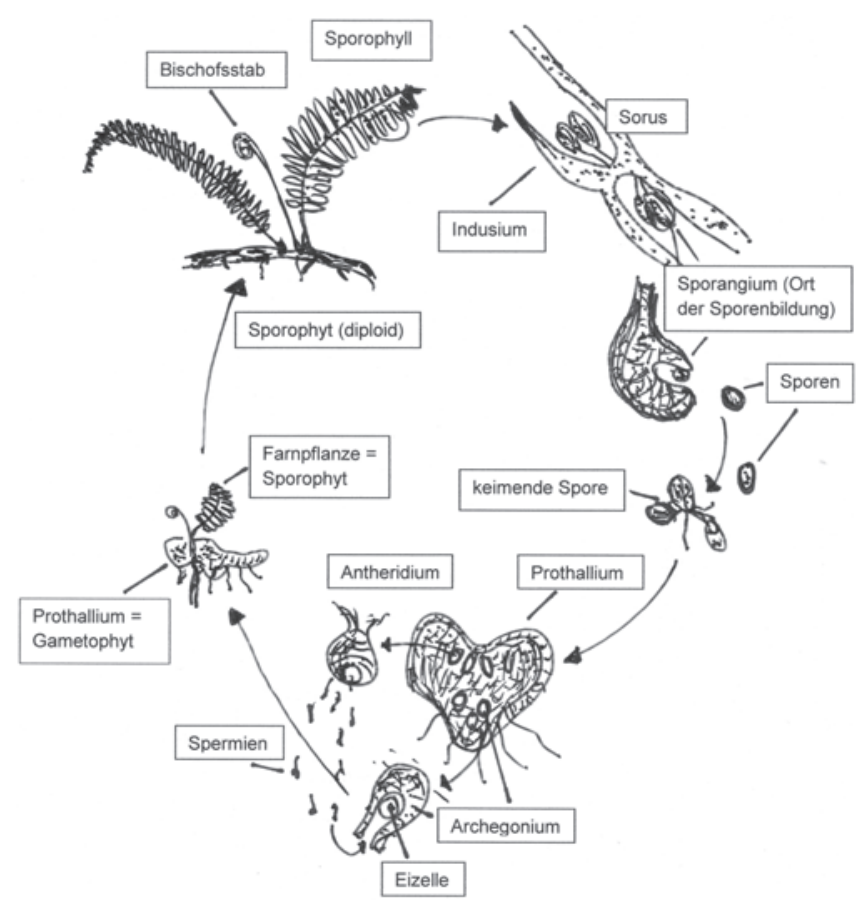

Abb. 11. Generationswechsel bei Farnen (Felix Gartmann, 2014).

Auch der Fürst der Botaniker, der grosse schwedische Naturforscher Carl von Linné, war sich sehr im Unklaren über die Natur der Gefässkryptogamen. In Briefen an den Schweizer Naturforscher Albrecht von Haller ist sich Linné nicht sicher, ob das «Mehl» bei Bärlappen die Samen oder Blütenstaub sind, um später zu behaupten, dass «Quod Lycopodii farina et capsulae sint flores vix dubito», also die Blüten, seien [8]!

\section{Generationenwechsel bei den Gefässkryptogamen}

Erst die Arbeiten des deutschen Botanikers Wilhelm Hofmeister brachten 1851 Licht ins Dunkel bezüglich der Vermehrung der Farne und ihrer Verwandten: «Moose und Farne bieten somit eines der auffälligsten Beispiele eines regelmässigen Wechsels zweier in ihrer Organisation weit verschiedener Generationen. Die ersten derselben, aus der keimenden Spore hervorgegangen, entwickelt Antheridien und Archegonien, bald wenige, bald viele. In der Centralzelle des Archegoniums entsteht in der Folge durch die aus den Antheridien entleerten Spermatozoidien die zweite Generation, bestimmt Sporen zu erzeugen, deren sie stets eine weit grössere Zahl bildet, als die erste Generation Anlagen zur Frucht trug» [9].

Die auffällige Generation bei den Gefässkryptogamen (Abb. 11) sind die Sporophyten, die in den Sporangien mittels Meiose Sporen mit einem halbierten Chromosomensatz produzieren. Diese Sporen werden vom Wind verfrachtet, landen und keimen bei günstigen Bedingun-
Abb. 12. Asplenium viride, Asplenium adulterinum und Asplenium trichomanes (Foto: Felix Gartmann, 2014).

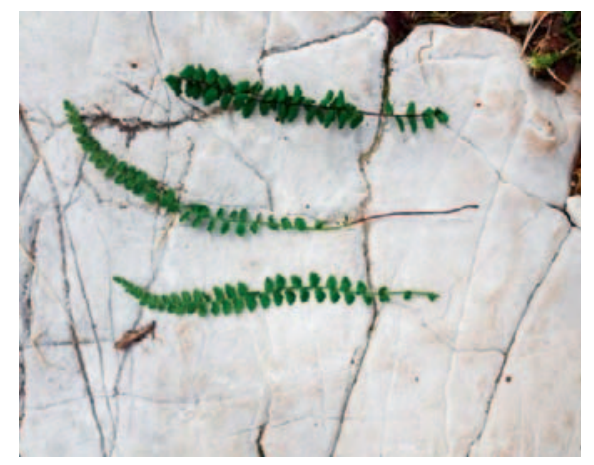

gen zur nächsten Generation aus - den unscheinbaren Prothallien. Auf ihnen sitzen die Geschlechtsorgane: Antheridien und Archegonien. Erstere produzieren Spermien, die zu den Eizellen in den Archegonien schwimmen und sie befruchten. Daraus entsteht wiederum die Generation der Sporophyten, d.h. je nach Gruppe die uns bekannten Farne, Schachtelhalme, Bärlappe usw. Kreuzungen zwischen den Arten kommen bei Farnen und Schachtelhalmen häufig vor. Ein auffälliges Beispiel ist der Bastard zwischen dem Braunstieligen Streifenfarn (Asplenium trichomanes L.) und dem Grünstieligen Streifenfarn (Asplenium viride HUDSON): der ehebrechende Streifenfarn (Asplenium adulterinum MILDE), dessen Name eher etwas über die Moralvorstellungen der beschreibenden Farnforscher als über die Biologie der beschriebenen Pflanzen aussagt (Abb. 12)!

Mit der Entdeckung der Fortpflanzungsweise der Farne und ihrer Verwandten verloren diese Pflanzen in $\mathrm{Eu}-$ ropa viel von ihrem romantischen Zauber. Niemand wird heutzutage mehr in der Johannisnacht nach Farnsamen suchen, um unsichtbar zu werden!

\section{Ethnobotanische Aspekte der Gefässkryptogamen}

Den ungefähr 250000 Samenpflanzenarten stehen nur ca. 14000 Arten von Gefässkryptogamen gegenüber. Schon rein quantitativ muss daher die ethnobotanische Bedeutung der Farne und ihrer Verwandten eher klein sein. Dazu kommt, dass diese Pflanzen weder über nährstoffreiche Samen noch über schmackhafte Früchte verfügen. Zudem sind viele Gefässkryptogamen leicht giftig und enthalten oft Bitterstoffe oder zähe, wenig geniessbare Gewebe. Das Fehlen von Blüten macht die Farne und ihre Verwandten auch für den Blumenhandel unattraktiv. Trotzdem spielen diese Pflanzen für Menschen verschiedener Kulturen eine gewisse Rolle als Bereicherung des Speisezettels, als Heilpflanzen, als Grundlage für Kosmetika, zur Gewinnung von Fasern und Farbstoffen sowie als Baumaterial. Ich habe mich bei der Auswahl der geografischen Räume von zwei Gedanken leiten lassen: In Regio- 
nen mit einer grossen Artenzahl an Gefässkryptogamen wie Indien, Papua-Neuguinea, tropischen Gebieten in Afrika und Südamerika sowie auf den Pazifischen Inseln liegt es nahe, dass einige dieser Pflanzen von den dort lebenden Menschen als Nähr-, Heil oder Zauberpflanzen genutzt werden. In arktischen Zonen, wo die Auswahl an nutzbaren Pflanzen für die dort lebenden indigenen Völker bescheiden ist, wurden (und werden?) möglicherweise in Ermangelung von Besserem auch Farne und ihre Verwandten gegessen oder als Medizinalpflanzen verwendet.

\section{Farne}

Farne traf und trifft man selten in den Kochtöpfen und Tellern dieser Welt an. Das geringe kulinarische Interesse an diesen Pflanzen zeigt sich auch daran, dass Laien Farne kaum nach Arten unterscheiden können. Eine Wandergruppe fragte mich während meiner pteridologischen Feldstudien im Engadin, worum es bei meiner Tätigkeit ginge. Auf meine Antwort, dass ich die Ökologie zweier alpiner Farnarten untersuche, kam die erstaunte Replik der Wanderer: «Was, gibt es denn zwei Farnarten?» Trotzdem wurden und werden Farne in verschiedenen Kulturen als Nahrungsmittel im kleinen Massstab verwendet. In Europa wurden die Rhizome von Adlerfarnen (P. aquilinium (L.) KUHN) - der Querschnitt des Blattstiels erinnert an einen Adler - während Hungersnöten gegessen [5]. Sonst ist die Verwendung von Pteridophyten als Lebensmittel auf unserem Kontinent in Vergessenheit geraten - mit der kleinen Ausnahme, dass die Rhizome des Engelsüss (Polypodium vulgare L.) mit ihrem an Lakritze erinnernden Geschmack als bescheidene Schleckerei gekaut werden können. In Ostasien gelten die Bischofsstäbe der Adlerfarne und von verwandten Arten als Delikatesse und werden ähnlich wie Spargel zubereitet [10], ebenso in Nigeria [11] und im Kongo [12]. Indigene Völker in den südwestlichen USA sammeln die Rhizome der Adlerfarne als Stärkespeicher, ähnlich wie bei uns die Kartoffeln [13]. Im östlichen Teil von Kanada und Neuengland sammelt man die frisch ausgerollten Blätter des Straussenfarns (Matteuccia struthiopteris (L.) TOD.), um sie als Gemüse zu essen [14]. Bei den Ureinwohnern in Nordostamerika werden Teile der Rhizome des blassgrünen Wurmfarns (Dryopteris expansa (C. PRESL) C.R.FRASER-JENK. \& JERMY) ähnlich wie Süsskartoffeln zubereitet, nach denen sie auch schmecken sollen [15]. Problematisch ist allerdings der Gehalt an potenziell krebserregenden Stoffen in diesen Pflanzen, sodass vor einem allzu extensiven Verzehr solcher Pteridophytenarten gewarnt wird [16-18].

In Hawaii wurden bis in die Mitte des 20. Jahrhunderts Sadleria cyatheoides KAULF. und verschiedene Arten des Baumfarns (Cibotium spp.) ihres Stärkereichtums wegen verwendet. Bischofsstäbe von Athyrium meyenianum MILDE und Christella cyatheoides (KAULF.) HOLTTUM wurden von den dortigen Inselbewohnern roh gegessen
Abb. 13. Marsilea quadrifolia (Foto: Felix Gartmann, 2014).

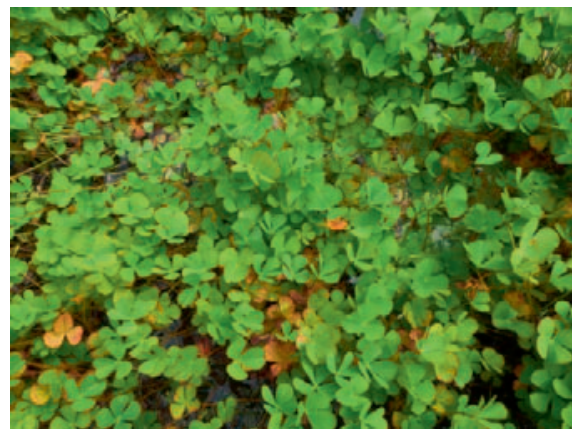

[19]. Auch auf anderen pazifischen Inseln werden die jungen Wedel verschiedener Farnarten manchmal ähnlich wie Spargeln gekocht, z.B. Acrostichum aureum L., Asplenium nidus L. (Nestfarn), Microsorum scolopendria (BURM.) COPEL., Tectaria spp. sowie Thelypteris spp. [20]. Im südlichen Nigeria werden zu diesem Zweck Botrychium lanuginosum WALL. ex HOOK. \& GREV. verwendet [11]. In Papua-Neuguinea werden Farnwedel zum Einpacken von Lebensmittel gebraucht, aber auch hier finden verschiedene Farnarten als Bischofsstäbe in der Küche Verwendung [21]. Die kugelförmigen Sporangienbehälter der Kleefarnart Marsilea drummondii A.BRAUN werden von den Aborigines Nordaustraliens zu einer Paste zerrieben («Nardoo»), die zur Brotherstellung dient [22]. Da aber diese Pflanze (Abb. 13) das ThiaminaseEnzym enthält, das zu einem Abbau von Vitamin B1 (Thiamin) führt, kann der Verzehr von unsachgemäss hergestelltem Nardoo zur Mangelkrankheit Beri-Beri führen. Die beiden Leiter der transaustralischen Expedition im Jahre 1860, Robert O'Hara Burke und William John Wills, kamen wegen dieses Marsilea-Produkts und des daraus folgenden Thiaminmangels ums Leben [23].

Als zeitgebundenes Kuriosum sei erwähnt, dass der amerikanische Botaniker Copeland 1942 den Anbau von Ceratopteris siliquosa (L.) COPEL. als Gemüse auf den Philippinen empfahl, wobei er ganz im Stil eines kolonialen Plantagenbesitzers Aufwand und Ertrag dieser Farnzucht vorrechnete: "Harvesting might cost half of the gross return. Obviously, it is work for women and children in spare time» [24]. Als eher indirekte Form der Pteridophytennutzung in der Landwirtschaft ist die Verwendung der Schwimmfarngattung Azolla LAM. (Abb. 14) zu nennen. Sie ist mit den stickstofffixierenden Cyanobakterien Anabaena azollae und Nostoc azollae vergesellschaftet und wird dadurch im Reisanbau in Ostasien als Gründünger verwendet [4].

Die Farne spielen als artenreichste Gruppe der Gefässkryptogamen in der Volksmedizin verschiedener Kulturen eine nicht unbedeutende Rolle. Beim Gemeinen Wurmfarn (D. filix-mas (L.) SCHOTT) deutet der Name auf die volksmedizinische Bedeutung hin. «Sein Wurzel gepulvert / unnd mit honig in einer Latwerge gestalt ein- 
Abb. 14. Azolla filicupflanzen (Foto: Felix Gartmann, 2014). loides zwischen Reis-

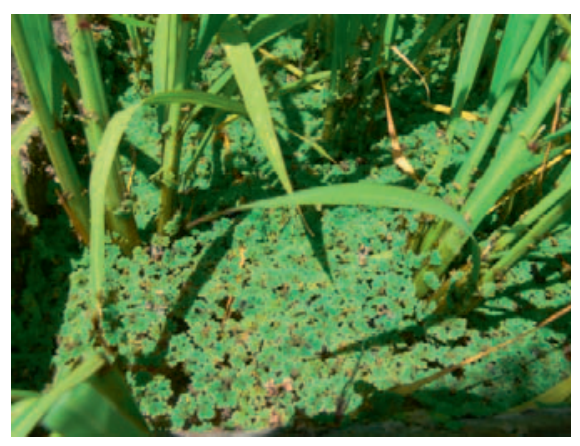

genommen / treibt auss die breyten würm» heisst es schon bei Fuchs [7]. In Nigeria wird die Pflanze für den gleichen Zweck eingesetzt [11]. Da es aber aufgrund des schwankenden Gehalts der Wirkstoffe in den Rhizomen schwierig ist, die korrekte Dosis zu finden, wurde dieses volksmedizinische Anthelminthikum durch andere, einfacher handhabbare und standardisierte Präparate ersetzt.

In der nordspanischen Provinz Kantabrien wird der Königsfarn (Osmunda regalis L.) bis in die Gegenwart als kommerziell genutzte Heilpflanze bei Knochenbrüchen, Gelenkschmerzen und rheumatischen Erkrankungen verwendet [25].

Die schon erwähnte Mondraute (B. lunaria (L.) SW.) wird in Indien als Wundheilmittel und gegen die Ruhr eingesetzt [26]. Im östlichen Norwegen wurde diese Art sowohl zur Heilung äusserlicher Wunden als auch bei Menstruationsbeschwerden verwendet [5]. In Böhmen wandte man die Mondraute neben der Wundheilung auch als Zauberpflanze zum Schutz des Viehs sowie als magisches Mittel an, auf dass die Geliebte wieder zurückkehren sollte. Die Alchemisten glaubten, mittels der Mondraute, die ihrer Ansicht nach in der Nacht wie der Mond leuchtet, unedle in edle Metalle verwandeln zu können, so beispielsweise in Conrad Gessners Arbeit: «De raris et admirandis herbis, quae sive quod noctu luceant, sive alias ob causas lunariae nominantur» aus dem Jahre 1555 [27]. Seltsam ist nur, dass Gessner durch einfache Beobachtung der Mondraute selbst auf die Idee hätte kommen können, dass es mit dem nächtlichen Leuchten dieser hübschen Farnart nicht weit her ist. Die Naturwissenschaften waren eben damals erst am Herandämmern ...

Das Venushaar (A. capillus-veneris L.) (Abb. 15) wird in verschiedenen Regionen Indiens volksmedizinisch als Aphrodisiakum sowie als Mittel gegen Menstruationsstörungen und zur Einleitung der Geburt benutzt. Besonders in den Western Ghats schätzen die Einheimischen Anwendungen des Venushaars als Stimulantium, als fiebersenkendes Mittel, gegen Verstopfung, als Juckreiz minderndes Präparat sowie als Haarwasser [28]. Bei den Tribals von Assam werden Extrakte dieser Pflanze bei Zahnschmerzen verwendet [29]. Die Pachmarhi in Zentralindien legen das Venushaar unter die Betten von Kin-
Abb. 15. Adiantum capillus-veneris (aus Thomé OW: Flora von Deutschland, Österreich und der Schweiz. 1885).

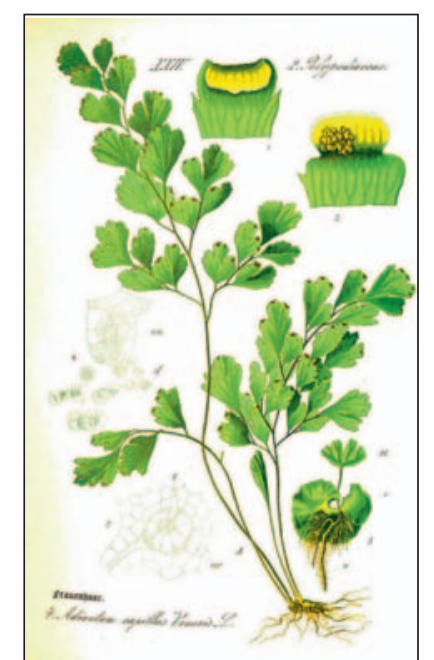

dern, um die Windpocken zu verhindern [30]. Auf dem ganzen indischen Subkontinent ist das Dekokt von A. capillus-veneris ein beliebtes Husten- und Bronchienmittel [26]. In Nepal wird diese Pflanze bei Kopfschmerzen auf die Stirn gedrückt. In Amazonien gilt ein aus dem Venushaar gekochter Sirup als harntreibend, hustenstillend sowie atemfördernd und wirkt gegen Menstruationsstörungen, Rheuma, Nierenstörungen, Gallensteine, saures Aufstossen sowie gegen Haarausfall [26]. Letzteres könnte ein Ausdruck der Signaturlehre sein, wonach das Aussehen von Heilpflanzen auf ihren Verwendungszweck hinweist, da die dünnen, dunklen Blattstiele an Frauenhaare erinnern. Im Kosovo verwendet die Landbevölkerung Venushaar gegen saures Aufstossen, Bronchitis sowie als Expektorans [31]. Im südlichen Nigeria verwenden Heiler Dekokte von A. capillus-veneris gegen Scheidenpilze. Extrakte der Rhizome dienen dort als Mittel gegen Schuppen [11]. In Französisch-Polynesien wird das Venushaar als Hustenlöser, fiebersenkendes Mittel, Hautlotion und Diuretikum benutzt [32]. Getrocknete Blätter des Venushaars werden in Lesotho als Heilmittel gegen Erkältungen geraucht [33]. In den Pyrenäen wird diese Art als blutstillendes Mittel, gegen Lungenentzündung und in hoher Konzentration zur Abtreibung verwendet [34]. Eine andere Art dieser Gattung, Adiantum lunulatum BURM. f. wird in Süd-Indien als Mittel gegen so unterschiedliche Erkrankungen wie Husten, Epilepsie, Harndrang und sogar Elephantiasis verwendet [35].

Trotz seiner Giftigkeit spielt der Adlerfarn (P. aquilinium (L.) KUHN) (Abb. 16) in verschiedenen Regionen dieser Welt eine gewisse Rolle als Heilpflanze. Neuere Studien zeigen eine antioxidative Wirkung von PteridiumExtrakten und ein damit verbundenes mögliches pharmazeutisches Potenzial [36]. Das Rhizom des Adlerfarns wird bei der Landbevölkerung von Madhya Pradesh in Zentralindien als Aphrodisiakum verwendet [37]. Im indischen Orissa trinken die Einheimischen einen Tee aus 


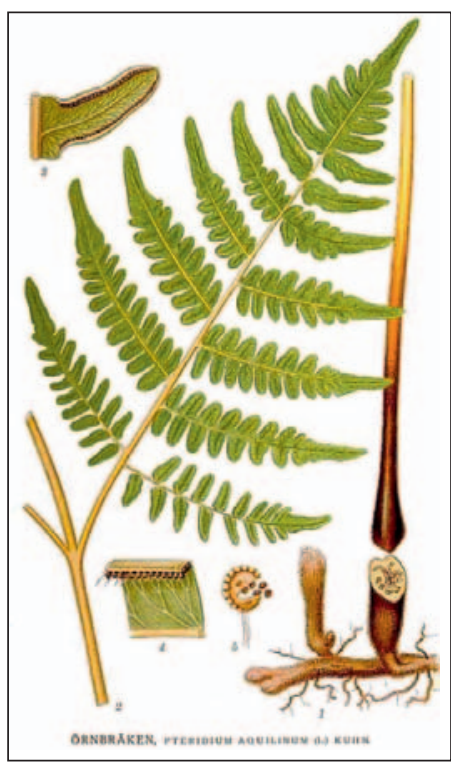

(aus Lindman CAM: Bilder ur Nordens Flora. 1905).

den Wedeln dieser Farnart gegen Bauchkrämpfe [38]. Im Kosovo gilt dieser Tee als harntreibend [31]. In PapuaNeuguinea dient der Saft der Blattstiele von Pteridium als Mittel gegen Zahnschmerzen und bei Infektionen der Mundhöhle [22]. Die Bomagai im gleichen Gebiet legen Adlerfarnwedel um ihre Häuser, um Krankheiten am Eindringen zu hindern [21]. In Nigeria ist bei der Landbevölkerung ein aus den Pteridium-Rhizomen hergestellter Gesundheitstee sehr geschätzt [11]. In den Pyrenäen wird ein Absud der Wedel als harntreibendes Mittel eingenommen. In dieser Region wird ein Tee aus den Wedeln von $P$. vulgare L. als Blutdrucksenker verwendet, wobei die Anzahl der Sori auf den verwendeten Blättern Auskunft über die Qualität des Heilmittels gibt [32]. Bei der Urbevölkerung von Alaska werden Dekokte von A. filix-femina gegen Augenkrankheiten, Nierenstörungen und Tuberkulose verwendet [39]. In Ost-Timor werden Tees aus den Wedeln von Drynaria quercifolia (L.) J. SM. von Heilern gegen Halsentzündungen eingesetzt [40].

Farne finden jedoch auch in der Gegenwart medizinische Anwendung. Oral verabreichte Extrakte der neotropischen Farnart Phlebodium aureum (L.) J.Sm. haben einen heilenden Effekt bei Sonnenbrand [41], während $L y$ godium flexosum (L.) SW. eine antibakterielle Wirkung gegen multiresistente Erreger von Harnwegs- und Darmerkrankungen zeigt [42]. Eine ähnliche Wirkung gegen pathogene Bakterien besitzen Extrakte von Cyclosorus interruptus (WILLD.) H. ITO [43]. Methanolische Blattextrakte des tropischen Baumfarns Cyathea gigantea (WALL. ex HOOK.) HOLTTUM zeigen im Tierversuch eine Ödem lindernde Wirkung [44]. Gegen Husten wirken Extrakte aus Marsilea minuta L., die zudem schleimauswurffördernd sind [45]. Bei einer Reihe indischer Farnarten haben deren Extrakte eine Wirkung gegen die pathogene gramnegative Bakterienart Xanthomonas cam- pestris gezeigt [46]. Bei Tectaria coadunata (WALL. ex HAINES) RAIZADA \& N.P.CHOWDHURY, einem Farn, der in Ostasien zur Behandlung von Insektenstichen verwendet wird, konnten kürzlich einige entzündungshemmende Wirkstoffe identifiziert werden [47].

\section{Bärlappe}

Der Keulenbärlapp (Lycopodium clavatum L.) hatte dank seiner enormen Sporenproduktion von allen Bärlappgewächsen schon früh die Aufmerksamkeit der Menschen gewonnen. So wurden die gelblichen, sich fettig anfühlenden Sporen als Wund- und Babypuder verwendet, z.B. bei westamerikanischen indigenen Völkern [13] und bei Bewohnern Nordeuropas [5], aber auch als Brechmittel bei Vergiftungen in Papua-Neuguinea, auf den Philippinen [22] und in Indien [48]. In der Slowakei wurden Dekokte von Bärlappen in der Volksmedizin als Abortivum eingesetzt, daher die lokale Bezeichnung für Bärlappe: Netata $=$ «nicht Vater», d.h. kein Kind! Im südlichen Afrika werden getrocknete Bärlapp-Pflanzen als Mittel gegen Erkältungen geraucht [33], in Nigeria werden Absude des Keulenbärlapps zur Wunddesinfektion appliziert. Zudem dienen Extrakte von Huperzia selago (L.) BERNH. ex SCHRANK \& MART. (Tannenbärlapp) in diesem Land als Gegengift bei Schlangenbissen [11]. Bei dieser Art wurden antioxidative Substanzen gefunden, die eine pharmazeutische Wirkung plausibel machen [36]. Bei den First Nations in Kanada wurden Lycopodium lucidulum MICHX. und H. selago gegessen [49], was sehr aussergewöhnlich ist, da die meisten Bärlappe eine Textur haben, die an Tannenzweige erinnert und somit kaum kulinarische Genüsse verheisst. Auch in der Kriminalistik haben Bärlappsporen Einzug gehalten: Wegradierte Bleistiftnotizen können mit ihnen wieder sichtbar gemacht werden [50]. Die Anwendung dieser Sporen ist allerdings mit Problemen verbunden. So haben mit Bärlappsporen bepuderte Latexhandschuhe für medizinische Zwecke bei Patienten zu chronischen postoperativen Entzündungen geführt [51], oder mit diesen Sporen behandelte Kondome führten zu allergischen Reaktionen [52].

\section{Moosfarne}

Trotz ihrer äusserlichen Unscheinbarkeit haben Selaginella-Arten eine gewisse ethnomedizinische Bedeutung erlangt. In Nordost-Brasilien wird Selaginella convoluta (ARN.) SPRING von den Indigenen als Dekokt oder Mazerat als Aphrodisiakum, als Diuretikum sowie gegen Menstruationsstörungen eingesetzt [53]. In Zentralindien spielen Moosfarne als Heilpflanzen in der traditionellen Medizin eine gewisse Rolle.

So werden Pasten aus Selaginella bryopteris (L.) BAKER gegen Gonorrhö eingesetzt und Dekokte von Selaginella ciliaris (RETZ.) SPRING sowie Selaginella repanda (DESV. ex POIR.) gegen Störungen der Monatsblutung 


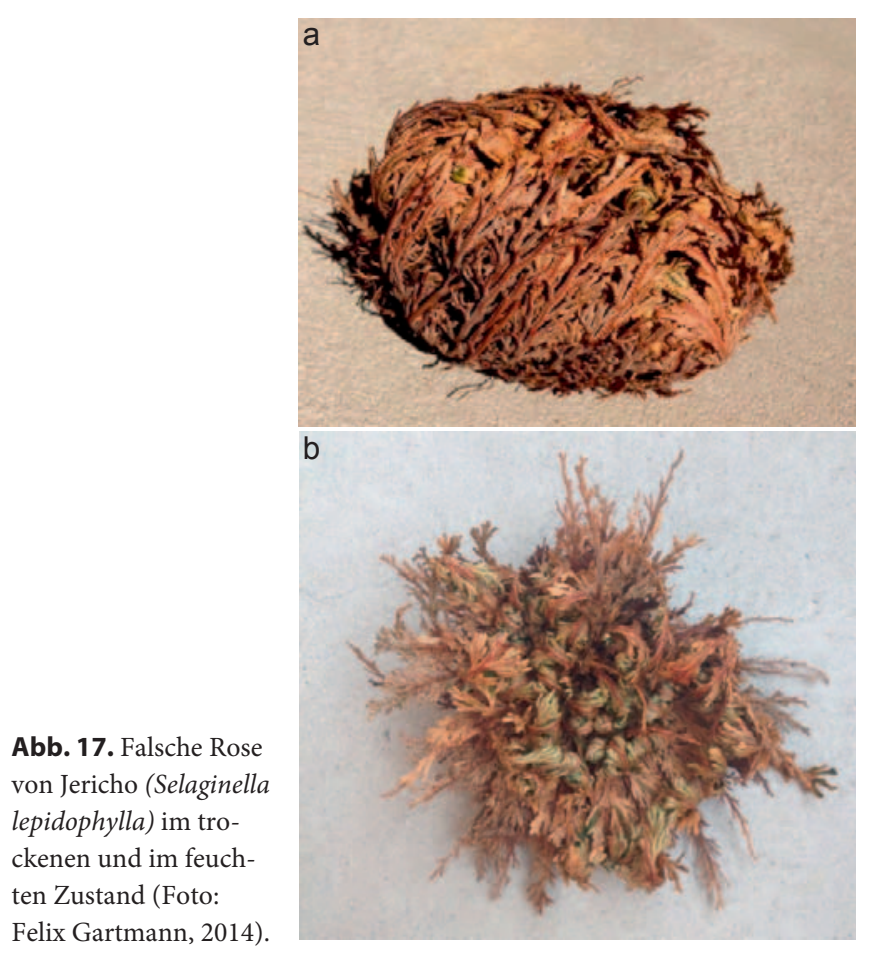

verwendet [37] - dies gilt ebenso für Papua-Neuguinea (Selaginella flabellata (L.) SPRING) [21]. Bei den Mbuti und Ewe im Kongo werden Hautkrankheiten mit Moosfarnblättern behandelt [54]. S. repanda (DESV. ex POIR.) wird in Orissa, Indien, als Paste gegen die Hautpusteln der Lepra eingesetzt [38]. Extrakte aus den Blättern von Selaginella wallichi (HOOK. \& GREV.) werden von Wöchnerinnen in der indischen Region von Melghat als Tonikum verwendet [48].

Auf Java werden Moosfarne gegen Amenorrhö verwendet, darüber hinaus auch zur Wundheilung, gegen Blut im Stuhl sowie gegen Hämorrhoiden. Dekokte aus Selaginella werden aber auch zur allgemeinen Stärkung der Gesundheit und für ein langes Leben eingesetzt [55]. In den Arawali-Bergen in Indien finden sich vergleichbare Anwendungen für S. bryopteris (L.) BAKER. Im Glauben der dortigen Bevölkerung ist es sogar möglich, mit dieser Pflanze Tote wiederzubeleben [56]. Möglicherweise hängt dies mit der Fähigkeit einiger Moosfarne zusammen, längere Trockenzeiten zusammengerollt zu überleben, um sich bei erneutem Regen zu entrollen. Die auch in unseren Regionen bekannte "Falsche Rose von Jericho" (Selaginella lepidophylla (HOOK. \& GREV.) SPRING) (Abb. 17) zeigt dieses Verhalten auffällig und wird in Deutschland als Symbol für die Auferstehung Christi als «Auferstehungsfarn» gehandelt.

Pharmakologische Untersuchungen von S. lepidophylla (HOOK. \& GREV.) SPRING zeigen, dass der allgemeine Gebrauch dieser Pflanze in Asien als antitumorales und entzündungshemmendes Phytotherapeutikum auf ihrem Gehalt an Biflavonoiden gründet [57].

\section{Schachtelhalme}

Die Equisetum-Arten mit ihrem besonderen Habitus werden auch von Laien leicht erkannt, wenn auch nicht auf Artniveau. Fatal können Verwechslungen zwischen dem Ackerschachtelhalm (Equisetum arvense L.), der als Heilpflanze verwendet wird, und dem giftigen Sumpfschachtelhalm (Equisetum palustre L.) sein. Schachtelhalme wurden früher bei uns dank ihres Gehalts an Kieselsäure als Scheuermittel für Zinngeschirr verwendet, daher auch der Name Zinnkraut. Bei den indigenen Völkern Nordamerikas erfreute sich die Gattung grosser Wertschätzung: Die Schachtelhalme dienten als Poliermittel für Pfeile und Bögen, aber auch als Nahrungsmittel. So wurden junge fertile Stängel von E. arvense bei den ChinookIndianern geschält und roh gegessen. Equisetum laevigatum A. BRAUN in der gleichen Form diente dem HopiVolk vermischt mit Maismehl als Bestandteil eines Breis, der auch für zeremonielle Zwecke verwendet wurde [13].

Die Inuit-Völker Kanadas nutz(t)en verschiedene Schachtelhalmarten als Nahrung, manchmal roh mit Seehundfett vermischt [49]. Bei der Urbevölkerung im Nordosten der USA wurde Equisetum telmateia EHRH. (Riesenschachtelhalm) roh und gekocht gegessen [15]. Der Autor warnt allerdings vor dem Verzehr der sterilen Sprosse und empfiehlt: «(...) to be safe, eat the fertile shoots only in small quantities with extreme caution».

Als Heilpflanzen waren Schachtelhalme bei verschiedenen Kulturen sehr beliebt. Fuchs beschreibt die Wirkung des «Schaffthew» (= Schaftheu, d.h. Kastenheu) so: «Das Kraut in Wasser oder in Wein gesotten unnd getruncken / bekompt wol denen so blut speien / den frawen so zu seer fliessen» [7]. Getrocknete und gemahlene Pflanzen von Equisetum hyemale wurden beim Chinook-Volk in Nordamerika als Therapeutikum gegen Prostatavergrösserung verwendet [13]. Die Inuit von Alaska gebrauchen die Asche von E. arvense gegen Zahnschmerzen [39]. In Indien wird Equisetum ramosissimum DESF. traditionell als Dekokt gegen Gonorrhö eingesetzt und um die Fruchtbarkeit der Frauen zu verbessern [37]; Letzteres gilt auch für Lesotho [33]. Zudem wird die Pflanze als Salbe gegen Wundschorf und andere Hautläsionen verwendet. Auch zur Heilung von Knochenbrüchen wird diese Salbe von Bewohnern im indischen Orissa angewandt [38]. Im Kosovo gilt ein Tee aus E. arvense als nierensteinauflösend [31], ebenso in den Pyrenäen. Dort hat der Schachtelhalm ein hohes Ansehen als Heilpflanze bei vielerlei Beschwerden, z.B. gegen Entzündungen der Augen, der Ohren und der Nase, um den Blutkreislauf zu stärken, gegen Magengeschwüre und um den Appetit zu fördern [34].

\section{Brachsenkräuter}

Die in Europa seltenen Brachsenkräuter (I. lacustris L. und Isoëtes setacea LAM.) haben einen geringen Nutzwert 
für uns. Das verschleierte Brachsenkraut (Isoëtes velata A. BRAUN) wird als Aquarienpflanze gehandelt [58]. Sporen von Isoëtes coromandelina L. und Isoëtes panchananii D.D.PANT \& G.K.SRIVAST. werden in Indien in Medaillons als Schutz vor bösen Geistern getragen [37, 59].

\section{Urfarne}

Die sehr einfach gebauten Urfarne (Psilotum nudum (L.) P. BEAUV. und P. complanatum SW.) werden von uns kaum genutzt, da sie aufgrund ihrer Kleinheit und dem Mangel an interessanten Stoffen kaum je in das Bewusstsein der hiesigen Menschen gedrungen sind. Auf Hawaii wurden aus den ganzen Pflanzen von $P$. nudum Dekokte als Abführmittel und gegen Mundsoor bei Kindern verwendet; die Sporen wurden zudem als Babypuder gebraucht [19]. Auf der gleichen Inselgruppe wurde diese Urfarnart von Kindern als Spielzeug genutzt: Wessen Pflanze beim Biegen zuerst bricht, der hat den Zweikampf verloren [60]! Auf der Insel Moorea, Französisch-Polynesien, wird der Urfarn gegen Verstopfung und bei Zahnschmerzen eingesetzt [32], in der Traditionellen Chinesischen Medizin zudem gegen Menstruationsstörungen. Ein Dekokt von P. nudum, gemischt mit Kurkuma, wird in Indien als Paste gegen Hautläsionen verwendet. Antibakterielle Wirkstoffe in dieser Pflanze wurden nachgewiesen [61].

\section{Schlussbetrachtung}

Die Farne und ihre Verwandten (Schachtelhalme, Bärlappe, Moosfarne, Brachsenkräuter und Urfarne) stellen mit rund 12000 Arten im Vergleich zu den Samenpflanzen, von denen es rund 226000 Arten gibt, eine "Quantité négligeable» dar. Dies trifft aber nicht im gleichen Mass für die Bedeutung der Gefässkryptogamen als Nutzpflanzen, insbesondere als Heilkräuter, zu. Verzehrt werden diese Pflanzen zwar selten, da sie oft bitter schmecken, giftige Inhaltsstoffe enthalten, über sehr zähe Festigungsgewebe verfügen und die Schachtelhalme zudem Kieselsäurekristalle in ihren Zellen einbauen. Aus diesen Gründen wurden Farne in Europa nur während Hungersnöten als Nahrung verwendet. In Ostasien und bei Ureinwohnern der kalten Gebiete werden Pteridophyten hingegen regelmässig konsumiert - dies trotz der Bedenken von Wissenschaftlern, die wiederholt auf die mögliche krebserregende Wirkung einiger Inhaltsstoffe aufmerksam machen.

In der Ethnomedizin spielen die hier untersuchten Pflanzengruppen eine gewisse Bedeutung, die mit der Artenzahl an bestimmten Orten zu korrelieren scheint. Indien mit seinen 1042 Pteridophytenarten und Neuguinea mit 1183 Arten sind Hotspots der pharmazeutischen Nutzung dieser Pflanzen. Auch Hawaii mit seinen 167 Gefässkryptogamenarten auf einer Gesamtfläche von $16729 \mathrm{~km}^{2}$ tritt in der ethnomedizinischen Literatur prominent auf. Es fällt auf, dass sich die Nutzung von einzelnen Arten in verschiedenen Kulturen gleicht. Beispielsweise werden Moosfarne (Selaginella spp.) in Indien, auf Java und in Brasilien gegen Menstruationsstörungen eingesetzt. Die Schachtelhalmart E. ramosissimum wird in Indien und in Lesotho zur Förderung der Fruchtbarkeit von Frauen eingesetzt, ein Absud von E. arvense hilft sowohl im Kosovo als auch in den Pyrenäen gegen Nierensteine. Der Urfarn P. nudum wurde sowohl in Hawaii als auch auf der über $4200 \mathrm{~km}$ entfernten Insel Moorea gegen Zahnschmerzen eingesetzt. Die Mondraute (B. lunaria) wurde in Norwegen, Böhmen und Indien zur Wundheilung verwendet; das zierliche Venushaar (A. capillus-veneris) ist in Indien, im Amazonasgebiet und im Kosovo ein beliebtes Hustenmittel. In Europa scheint das Wissen um die Heilkräfte der Farne und ihrer Verwandten mit dem Siegeszug der biologischen Medizin verlorengegangen zu sein, nicht zu sprechen von den magischen Kräften der Pteridophyten wie unsichtbare Farnsamen und Widdertone. Studien belegen jedoch, dass in einigen der hier besprochenen Arten interessante Wirkstoffe zu finden sind. Bis jetzt nicht untersuchte Pteridophytenarten haben möglicherweise ein grosses pharmakologisches Potenzial. So gibt es auf Madagaskar fast 600 Farnarten, von denen 245 endemisch sind [62]. Es ist zu hoffen, dass die Einheimischen beim Heben dieser Schätze ihren Anteil an der Vermarktung der heilkräftigen Substanzen erhalten!

\section{Dank}

Besten Dank meiner Tochter Ladina für das sorgfältige Korrektorat und Maja Dal Cero für die Betreuung dieser Arbeit.

\section{Disclosure Statement}

Felix Gartmann ist Botaniker und Berufsschullehrer. Die vorliegende Arbeit ist die überarbeitete Version einer Abschlussarbeit des Zertifikatstudiengangs «Ethnobotanik und Ethnomedizin» der Universität Zürich.

Für den Autor bestehen keinerlei Interessenkonflikte.

\section{Supplemental Material}

Siehe Supplemental Material unter www.karger.com/doi/ 10.1159/000371460.

Tab. 1. Ethnobotanische Nutzung einiger Gefässkryptogamen 
1 www.magrama.gob.es/es/biodiversidad/temas/ conservacion-de-especies-amenazadas/catalogo-nacional-de-especies-amenazadas/peligro_extincion.aspx (zuletzt aktualisiert 2006, zuletzt geprüft am 14.09.2014)

2 Lorenz M: Okkulte Botanik. Farnkraut und Farnsamen. Zentralbl Okkult 1921/22;15: 265-267.

3 Bock H: Das Kreütter Buch. Strassburg, 1539.

4 Kramer KU, Schneller JJ, Wollenweber E: Farne und Farnverwandte. Morphologie, Systematik, Biologie. Stuttgart, Thieme, 1995.

5 Øllgaard B, Tind K: Scandinavian Ferns. A Natural History of the Ferns, Clubmosses, Quillworts, and Horsetails of Denmark, Norway, and Sweden. Copenhagen, Rhodos, 1993.

6 Corne FC: Ferns - facts and fancies about them - II. Am Fern J 1924;14:77-82.

7 Fuchs L: New Kreüterbuch. Band 1. Basel, 1543. 8 Linné C: Briefe an Albrecht von Haller. Linnaeus, Carl (12 Letters from Albrecht von Haller, Oct. 13, 1737 to Oct. 23, 1749, \& 1 letter from Theophilus Emanuel Haller, anno 1763, to Linnaeus). Analecta epistolarum, in primis historiam et res litterarias Sveciae illustrantium (...) (Uppsala, 1792), 20-23. Soulsby no. 2468. http://linnaeus.c18.net/Letter/L0185 (zuletzt geprüft am 05.08.2014).

9 Hofmeister W: Vergleichende Untersuchungen der Keimung, Entfaltung und Fruchtbildung höherer Kryptogamen. Leipzig, 1851.

10 Liu Y, Wujisguleng W, Long C: Food uses of ferns in China: a review. Acta Soc Bot Pol 2012;81:263-270.

11 Nwosu MO: Ethnobotanical studies on some pteridophytes of Southern Nigeria. Econ Bot 2002;56:255-259.

12 Van der Burgt X, Van der Maesen J, Onana J-M (eds): Systématique et Conservation des Plantes Africaines. Le Savoir-Faire Local dans la Valorisation Alimentaire de la Fougère (RD CONGO). Yaoundé, Cameroun, 2010, pp 333-339.

13 Lloyd R M: Ethnobotanical uses of California pteridophytes by Western Indians. Am Fern J 1964;54:76-82.

14 CBS Interactive Inc. (Hrsg): Rezept für Fiddlehead Fern. www.chow.com/ingredients/72 (zuletzt geprüft am 24.08.2014).

15 Clay-Poole S: Ethnobotany - Ferns and FernAllies. Washington State Department of Transportation (Olympia, WA, USA), Olympia. www.wsdot.wa.gov/environment/culres/ ferns.htm (zuletzt geprüft am 05.08.2014).

16 Evans IA, Widdop B, Jones RS, Barber GD, Leach $\mathrm{H}$, Jones DL, Mainwaring-Burton R: The possible human hazard of the naturally occurring bracken carcinogen. Biochem J 1971;124:29-30.

17 Vetter J: Toxicological and medicinal aspects of the most frequent fern species, Pteridium aquilinum (L.) Kuhn; in Kumar A, Fernández H, Revilla MA (eds): Working with Ferns. New York, Springer, 2010, pp 361-375

18 Bolton J: Facts on Fiddleheads. Orono, ME, University of Maine, 2013. http://extension. umaine.edu/publications/4198e/ (zuletzt geprüft am 05.08.2014).

19 Fosberg FR: Uses of Hawaiian ferns. Am Fern J 1942;32:15-23.
20 Thaman RR: Batiri Kei Baravi. The ethnobotany of Pacific island costal plants. Atoll Res Bull 1992;(361):1-62.

21 Milliken W: Ethnobotany of the Yali of West Papua. Royal Botanic Garden, Edinburgh. http://rbg-web2.rbge.org.uk/ethnobotany/yali $p d f$.

22 Croft J: Ferns and Man in New Guinea. Australian National Botanic Gardens. Canberra, 1982. www.anbg.gov.au/fern/ferns-man-ng htm (zuletzt aktualisiert am 1999, zuletzt geprüft am 05.08.2014).

23 Burke and Wills Expedition (2014). http:// en.wikipedia.org/wiki/Burke and Wills expedition\#Cause_of_death (zuletzt geprüft am 24.08.2014).

24 Copeland EB: Edible ferns. Am Fern J 1942; 32:121-126.

25 Molina M, Reyes-GarcÍa V, Pardo-de-Santayana $\mathrm{M}$ : Local knowledge and management of the Royal Fern (Osmunda regalis L.) in Northern Spain: implications for biodiversity conservation. Am Fern J 2009;99:45-55.

26 Srivastava K: Ethnobotanical studies on some important ferns. Ethnobot Leaflets 2007;11 164-172.

27 Kramer KU (Hrsg): Illustrierte Flora von Mitteleuropa. Band 1, Teil 1. Berlin, Parey, 1984.

28 Benniamin A: Medicinal ferns of North Eastern India with special reference to Arunachal Pradesh. Indian J Tradit Knowl 2011;10:516522.

29 Barukial S; Sarmah JN: Ethnomedical plants used in Golaghat, Assam. Int J Med Arom Plants 2011;1:203-211.

30 Singh BP, Upadhyay R: Ethno-botanical importance of Pteridophytes used by the tribe of Pachmarhi, Central India. J Med Plants Res 2012;6:14-18.

31 Mustafa B, Hajdari A, Krasniqi F, Hoxha E, Ademi H, Quave CL, Pieroni, A: Medical ethnobotany of the Albanian Alps in Kosovo. J Ethnobiol Ethnomed 2012;8:6.

32 Baltrushes N: Medical Ethnobotany, Phytochemistry and Bioactivity of the Ferns of Moorea, French Polynesia. Dissertation. De partment of Integrative Biology, University of California, Berkeley, CA, USA. 2006. http:// ucjeps.berkeley.edu/moorea/Baltrushes2006.pdf.

33 Moteetee A, Van Wyk B-E: The medical ethnobotany of Lesotho. Bothalia 2011;41:209228.

34 Bonet MÀ, Agelet A, Vallès J, Villar L: Contribution à la connaissance ethnobotanique des ptéridophytes dans les Pyrénées les Pyrénées. Bocconea 2001;13:605-612.

35 Pallavi G: An ethno-pharmaco-botanical review of hamsapadi - Adiantum lunulatum Burm. F. (A. Philippense Linn.). Int J Pharma Biol Arch 2011;2:1627-1635.

36 Talukdar AD, Tarafdar RG, Choudhury MD Nath D, Choudhury S: A review on pteridophyte antioxidants and their potential role in discovery of new drugs. Assam University J Sci Technol 2011;7:151-155.

37 Singh S, Singh R: Utilization of pteridophytes of Achanakmar-Amarkantak Biosphere Reserve, Central India in women's health and beauty care practices. Int J Pharm Sci Rev Res 2013;4:235-240
38 Rout SD, Panda T, Mishra N: Ethnomedical studies on some pteridophytes of Similipal Biosphere Reserve, Orissa, India. Int J Med Med Sci 2009;1:192-197.

39 Garibaldi A: Medicinal flora of the Alaska natives. A compilation of knowledge from literary sources of Aleut, Alutiiq, Athabascan, Eyak, Haida, Inupiat, Tlingit, Tsimshian, and Yupik traditional healing methods using plants. Anchorage, Alaska, Alaska Natural Heritage Program, Environment and Natural Resources Institute, University of Alaska Anchorage,1999.

40 Collins SW, Martins X, Mitchell A, Teshome A, Arnason JT: Fataluku medicinal ethnobotany and the East Timorese military resistance. J Ethnobiol Ethnomed 2007;3:5.

41 Middelkamp-Hup MA, Pathak MA, Parrado C, et al: Oral Polypodium leucotomos extract decreases ultraviolet-induced damage of human skin. J Am Acad Dermatol 2004;51:910-918.

42 Nayak N, Rath S, Mishra MP, et al: Antibacterial activity of the terrestrial fern Lygodium flexuosum (L.) Sw. against multidrug resistant enteric- and uro-pathogenic bacteria. J Acute Dis 2013;270-276.

43 Pauline VC, Irudayaraj V, Johnson M: Antibacterial efficacy of macroscopic, microscopic parts of sporophyte and in vitro cultured gametophyte of a fern Cyclosorus interruptus (Willd.) H. Ito (Thelypteridaceae - Pteridophyta). J Chem Pharm Res 2012;4:1167-1172.

44 Madhukiran P, Rao GB: Anti-inflammatory activity of methanolic leaf extract of Cyathea Gigantea (WALL. EX HOOK.). Int J Pharm Res Dev 2011;3:64-68.

45 Chakraborty R, De Britto AJ, Devanna N, Sen S: Antitussive, expectorant activity of Marsilea minuta L., an Indian vegetable. J Adv Pharm Technol Res 2013;4:61-64.

46 Gracelin D, Herin S, De Britto A J, Kumar PB Jeya R: Antimicrobial potency of five south Indian ferns against Xanthomonas campestris. J Biopest 2012;5:196-200.

47 Dubal KN, Ghorpade PN, Kale MV: Studies on bioactive compounds of Tectaria Coadunata (Wall. ex Hook. \& Grev.) C. Chr. Asian Pharm Clin Res 2013;6(suppl 2):186-187.

48 Shirsat RP: Ethnomedicinal uses of some common lower plants used by tribals of $\mathrm{Mel}$ ghat region (MS), India. Ethnobot Leaflets 2008;667-669.

49 Kuhnlein H, Turner NJ: Traditional Plant Foods of Canadian Indigenous Peoples. Nutrition, Botany and Use. McGill Unniversity (Quebec), University of Victoria (Victoria), 2009. www. fao.org/wairdocs/other/ai215e/ AI215E06.htm (zuletzt aktualisiert am März 2009, zuletzt geprüft am 05.08.2014).

50 Welch JR: Lycode powders in a case of erasure. J Forensic Sci Soc 1982;22:43-44

51 Antopol W, Robbins C: Lycopodium granuloma resulting from the use of anal suppositories. JAMA 1937;109:1192.

52 Balick MJ, Beitel JM: Lycopodium spores used in condom manufacture: associated health hazards. Econ Bot 1989;43:373-377.

53 de Fatima Agra M, Baracho GS, Diniz Basílio IJ et al: Synopsis of the medicinal flora of 'CARIR PARAIBANO'. Oec Aus 2009; 11:323-330. www.oecologiaaustralis.org/ojs/index.php/oa/ar ticle/view/191 (zuletzt geprüft am 14.09.2014). 
54 Terashima H, Ichikawa M: A comparative ethnobotany of the Mbuti and Efe huntergatherers in the Ituri Forest, Democratic Republic of Congo. Afr Study Monogr 2003;24 $1-168$.

55 Setyawan AD: Traditionally utilization of Selaginella; field research and literature review. Nusantra Biosci 2009;1:146-158.

56 Reena A, Rini T: A mini review on medicinal properties of the resurrecting plant Selaginella bryopteris (Sanjeevani). Int J Pharm Life Sci 2011;2:933-939.
57 Li S, Yao H, Zhao M, et al: Determination of seven biflavones of Selaginella Doederleinii by high performance liquid chromatography. Analytical Letters 2013;46:2835-2845

58 Isoetes velata var. sicula (Verschleiertes Brachsenkraut) als Aquariumpflanze. www. flowgrow.de/db/wasserpflanzen/isoetes-velatavar-sicula (zuletzt geprüft am 05.08.2014).

59 Singh BP, et al: Ethnobotanical uses of Isoëtes coromandelina L.F. and Actinopteris radiata (SW.) at Aravali Hills Rampura Region. Life Sci Leaflets 2013. http://lifesciencesleaflets. ning.com (zuletzt geprüft am 05.08.2014).
60 University of Hawaii: Psilotum nudum-Verwendung in Hawaii. 2009. www.nativeplants. hawaii.edu/plant/view/Psilotum_nudum (zuletzt geprüft am 05.08.2014)

61 Noreen A: 10 Medisinplanter fra Burma. En litteraturstudie. Masterarbeit. Pharmazeutisches Institut, Universität Oslo, Oslo. 2012. www.duo.uio.no/handle/10852/12064 (zuletzt geprüft am 05.08.2014).

62 Hassler M, Swale B: World fern statistics by country 2001. www.planta.cn/forum/files planta/paragraphoe_635.pdf (zuletzt geprüft am 15.09.2014). 